\title{
Neuroimaging in schizophrenia: what does it tell the clinician?
}

\author{
James Woolley \& Philip McGuire
}

\begin{abstract}
Neuroimaging has been used in clinical practice for over 30 years, but it is still perceived as rarely offering the psychiatrist much help in direct patient management. As newer imaging modalities are introduced (from computed tomography and positron and single photon emission tomography to magnetic and functional magnetic resonance imaging), the promise of imminent clinical utility is reawakened, only to fade as the innovation is shown to be another, albeit useful, research tool. The aim of this article is to update readers on some recent advances that are starting to align the research and clinical functions of neuroimaging. As imaging becomes more accessible and affordable there is real promise that both clinicians and patients will begin to benefit more directly.
\end{abstract}

This article continues the series introduced by Robin McCreadie's editorial 'Schizophrenia revisited' (McCreadie, 2004). Previous contributors to the series are Leask (2004), Rowlands (2004), Connolly \& Kelly (2005), Gopal \& Variend (2005), Kerwin \& Bolonna (2005) and (Singh \& Fisher, 2005).

Neuroimaging promises much. The immediacy and visual impact of a scan are appealing to patients, carers and doctors alike. It reduces the divide separating psychiatry from the rest of medicine, and appeals to medical practitioners who are trained to make diagnoses on the basis not just of history but also of objective investigations. This is especially true of magnetic resonance imaging (MRI), which has relatively few side-effects, good soft-tissue resolution and the ability to highlight real-time functional changes (when used in the form of functional MRI (fMRI)). However, to the practising clinician the promise remains just that. Neuroimaging is still perceived to lack everyday utility for psychiatry. Where is the diagnostic scan for schizophrenia? Can a scan help to predict who is likely to benefit most from the latest expensive antipsychotic, or reassure us that an older drug is just as good? Can neuroimaging even help us with the increasing number of patients who request a scan 'just to check everything is $\mathrm{OK}^{\prime}$, when we think that, even if the scan is, the patient may not be?

\section{Exclusion of 'organic' causes}

This is a common reason for requesting a scan in suspected schizophrenia and it is gaining acceptance as scanning (especially MRI) becomes more widely available and affordable. Between 5 and 10\% of patients with psychosis (depending on the study) will have an 'organic' disorder that underlies their symptoms, rather than a 'functional' psychosis such as schizophrenia or bipolar disorder (Falkai, 1996). Other disorders that can be associated with psychotic symptoms include epilepsy, brain injury, slow-growing tumours, demyelinating diseases and encephalitis. The treatment for these conditions (e.g. anticonvulsants or tumour resection) may be fundamentally different from that for the major psychoses. Although some organic disorders are associated with neurological signs that can be detected on clinical examination, $50-100 \%$ of patients with schizophrenia also have neurological soft signs (Dazzan et al, 2004), and referral to a neurologist usually results in a request that the patient has an MRI scan. Thus, it is increasingly considered good clinical practice to scan all patients presenting with psychosis for the first time (American Psychiatric Association, 2004) and this is now recommended in contemporary textbooks of clinical psychiatry (Weinberger \& Hirsch, 2003).

\footnotetext{
James Woolley is a clinical research fellow at the Institute of Psychiatry and an honorary specialist registrar at the Maudsley Hospital, London (Section of Neuroimaging, PO67, Institute of Psychiatry, Denmark Hill, London SE5 8AF, UK. Tel: 020 7848 0369; fax: 0207848 0956; j.woolley@iop.kcl.ac.uk). His research interests include neuroimaging in obsessive-compulsive disorder and early psychosis, and clinical interventions to reduce the impact of pre-psychotic symptoms. Philip McGuire is Professor of Psychiatry and Cognitive Neuroscience and Head of the Section of Neuroimaging at the Institute of Psychiatry, London. His research is focused on neurocognitive processes that are putatively defective in psychosis. He is also Clinical Director of Outreach and Support in South London (OASIS) and of the Voices Clinic, Maudsley Hospital, and an honorary consultant to the Lambeth Early Onset (LEO) service.
} 


\section{Radiological anomalies and abnormalities in schizophrenia}

Some radiological abnormalities reflect the presence of conditions that could account for psychosis, whereas others are more common in psychosis but are not themselves responsible for the disorder. A large cavum septum pellucidum, for example, is more common in patients with schizophrenia than in controls, but it is not a causal factor in the disorder (Kasai et al, 2004). Estimates of the incidence of such 'non-causal' anomalies range between 30 and 40\% (compared with 5-12\% in the general population), and it has been suggested that they are evidence of the aberrant neurodevelopmental processes thought to contribute to schizophrenia. Lubman et al (2002) found that $30 \%$ of patients with schizophrenia were reported by a neuroradiologist to have abnormal scans. Most required no further action, but 4 of 242 individuals benefited directly from neuroimaging, being referred for alternative treatment. Standard clinical radiology can also identify some of the major neuroanatomical changes associated with schizophrenia, such as ventriculomegaly (Fig. 1) and cortical atrophy. There is a good correspondence between the detection of these major features in research analyses and in clinical radiology (Lawrie

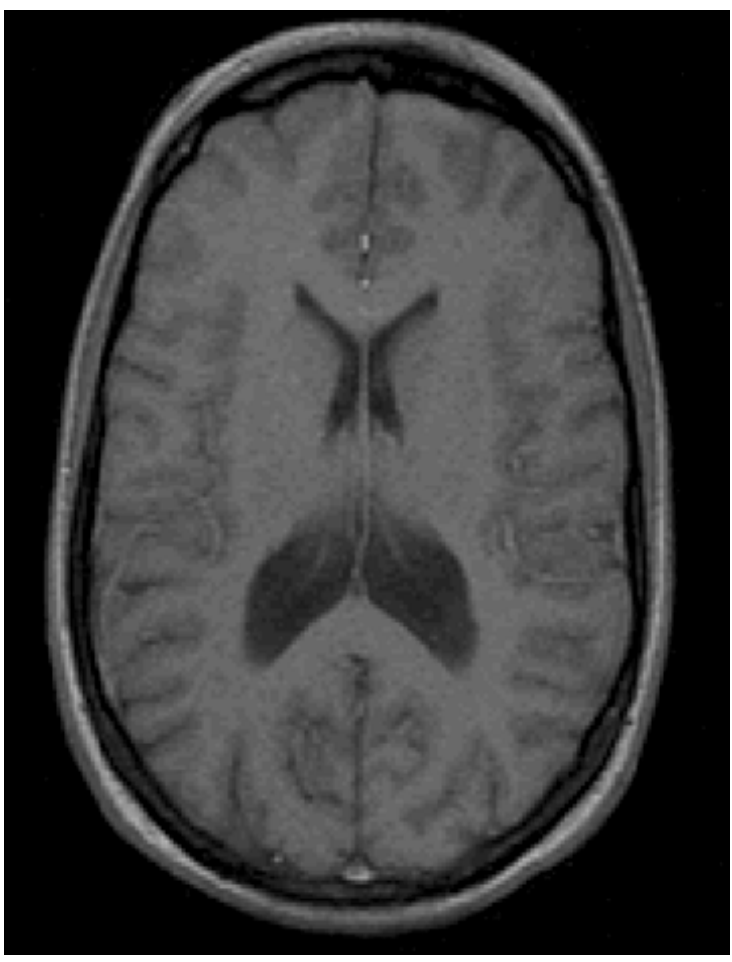

Box 1 Pathologies for which CT and MRI are useful screening tools

Computed tomography

- Stroke (haemorrhagic or old ischaemic)

- Tumours (large ones)

- Bleeding (intra- or extracranial)

- Volume loss

- Hydrocephalus

- Trauma

Magnetic resonance imaging

- Epilepsy

- Multiple sclerosis

- Certain tumours (e.g. pituitary adenomas)

- Vasculitis

et al, 1997), especially when standardised reporting methods are used (Smith et al, 1997).

\section{Cost-benefit analyses}

There is little published information on the costbenefit utility of neuroimaging in psychiatry. The cost of scanning is often perceived as expensive (typically £300-400 per patient), but this should be

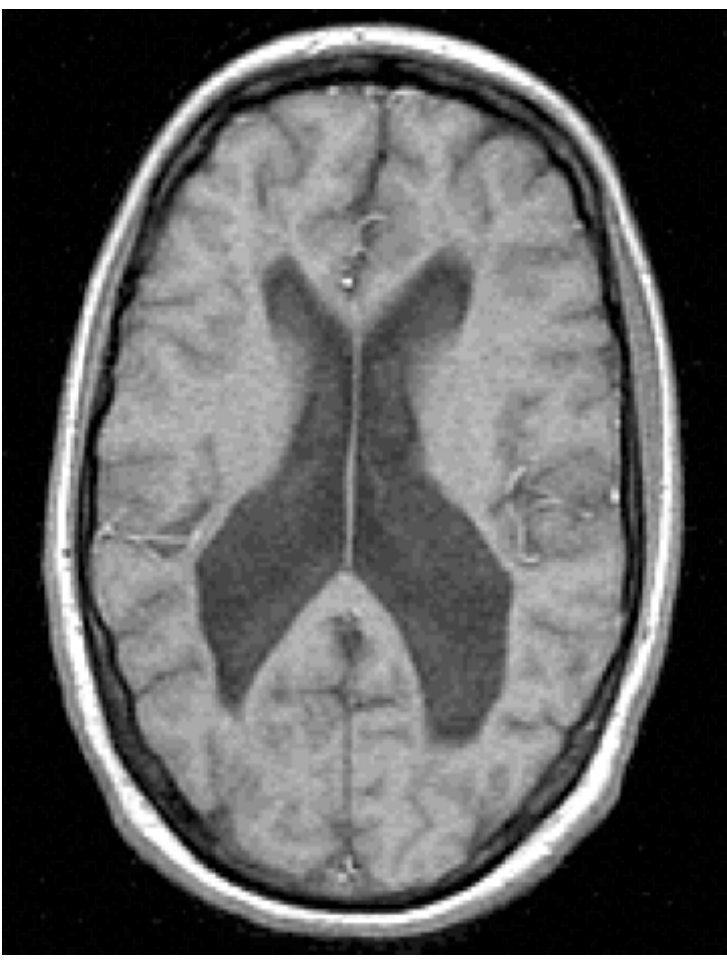

Fig. 1 Ventriculomegaly in discordant monozygotic twins seen on $T_{2}$-weighted MRI scans. Healthy twin (left) compared with twin with schizophrenia (right). With permission of Dr M. Picchioni. 
weighed against the cost of keeping patients in hospital while they are being assessed using clinical measures (of the order of $£ 200$ a day) and the costs of delaying appropriate treatment or providing inappropriate treatment. As with any investigation it is helpful to consider what is being looked for, how likely it is to be present, the costs involved and action depending on the result. The choice between the most commonly available types of scan - computed tomography (CT) and MRI - may depend on the nature of suspected pathology (Box 1). Computed tomography is cheap(er), quick and tolerable and can be used as a screening tool for psychiatric presentations. In patients with schizophrenia it is often considered in specific circumstances such as:

- the first presentation of psychosis (especially in unusual circumstances);

- rapid or unusual development of psychopathology;

- dementia;

- presence of focal neurological signs and symptoms.

However, the cost saving per scan for $\mathrm{CT}$ compared with MRI must be balanced against the risk of exposure to ionising radiation and of false-negative reports.

Magnetic resonance imaging is more expensive but provides much better image resolution and, because it does not involve $X$-rays, there are no risks or contraindications associated with exposure to radiation. In schizophrenia, MRI is indicated in the same circumstances as CT. However, it is better than $\mathrm{CT}$ at detecting some pathologies (Box 1).

\section{What else would help clinicians?}

Although there has been extensive research using structural and functional imaging in psychiatric disorders, there has been relatively little use of neuroimaging for purely clinical purposes in psychiatry (in contrast to its widespread use in the rest of medicine). Diagnosis and assessment of prognosis and effectiveness of treatments continue to be largely dependent on clinical history and current psychopathology. At present, neuroimaging does not to play a significant role in these areas, but there are signs that it might.

Since the landmark demonstration, using CT scanning, of enlarged ventricles in schizophrenia (Johnstone et al, 1976), a host of neuroimaging studies have identified areas of reduced grey matter in the disorder, including the hippocampus, parahippocampal gyrus, cingulate gyrus, insula, thalamus and the prefrontal and temporal cortex (Saykin et al, 1991). These structural studies have been complemented by functional imaging research which has indicated that schizophrenia is particularly associated with altered function in the prefrontal, cingulate and temporal cortex (McGuire \& Matsumoto, 2004) and that symptoms such as auditory hallucinations and thought disorder are mediated by these brain regions.

\section{Predicting course}

There is evidence that the severity of volumetric abnormalities at first presentation and their subsequent rate of progression are associated with a relatively poor prognosis in schizophrenia. In firstepisode patients, smaller cerebellum (Wassink et al, 1999), smaller Sylvian fissure and larger third ventricle (van Os et al, 1995), larger lateral ventricles (DeLisi et al, 1991) and ventriculomegaly and cortical atrophy (Zipursky et al, 1998a) have been found to be predictors of various measures of clinical and social outcome (Ho et al, 2003). More recent studies indicate that, although imaging findings at the time of the first episode may have some predictive value, the change within an individual's brain over time may be a better predictor of clinical outcome. Cahn and colleagues found that MRI abnormalities at baseline had little relation to subsequent course. However, the extent of grey matter loss, as revealed by comparison of follow-up and baseline scans, significantly correlated with clinical outcome (Cahn et al, 2002, 2004).

\section{Predicting response to treatment}

Neuroimaging may prove to be helpful in predicting which patients with schizophrenia are most likely to respond to treatment, especially in the first episode. Practical applications have been suggested for over a decade, with neuroimaging as part of a battery of tests (including baseline symptom severity, early reduction in symptom severity, initial subjective response to antipsychotic treatment, brain atrophy on neuroimaging and early changes in plasma homovanillic acid levels) at least partially predicting treatment outcome (Stern et al, 1993). Early studies suggested the predictive value of widely distributed brain changes such as average cortical sulcal width on CT scans (Schröder et al, 1993) or relatively increased grey matter volume on MRI (Zipursky et al, 1998b).

More recently, attempts have been made to make predictions in relation to more specific regions, but the results have been inconsistent. Clinical improvement with clozapine has been found in the presence of structural brain abnormalities such as wide Sylvian fissures, features previously associated with 
non-responsiveness to conventional antipsychotics (Lauriello et al, 1998). Similarly, Konicki et al (2001) found that patients with the greatest degree of functional improvement at follow-up had significantly less prefrontal sulcal widening on baseline MRI than those whose symptoms remained unchanged. In addition, there was no relationship between clozapine response and general sulcal widening.

Functional imaging studies, especially those using positron emission tomography (PET) to examine receptor occupancy, have been more directly useful, but it is difficult to apply their results to individual patients. They have illustrated the relationship between dopamine $D_{2}$ receptor occupancy, clinical response and side-effects, for example in first-episode schizophrenia (Kapur et al, 2000). Being able to predict that the likelihood of clinical response, hyperprolactinaemia and extrapyramidal sideeffects increases significantly as $\mathrm{D}_{2}$ occupancy exceeds specific thresholds facilitates the selection of the appropriate antipsychotic dose. $\mathrm{D}_{2}$ occupancy can also help explain many of the observed clinical differences between typical and atypical antipsychotics. PET could be used to establish the minimum dose that provides optimal $\mathrm{D}_{2}$ receptor occupancy (instead of titrating the dose according to clinical response and side-effects). However, this is impracticable because of the general unavailability of PET and single photon emission tomography (SPET), the cost (thousands of pounds per scan for PET) and the fact that many patients are too ill to scan. Consequently, this approach is mainly used in research, for example in evaluating the optimal dose of a new antipsychotic.

Neuroanatomy may predict response to electroconvulsive therapy (ECT), with a greater thirdventricle-to-brain ratio correlating with a larger number of treatments required to achieve benefit (Dequardo et al, 1997).

\section{Dose and drug selection}

Neuroimaging, and in particular functional techniques such as PET and SPET which measure neuroreceptor occupancy in vivo (Fig. 2), has bolstered the scientific rationale for moving away from high-dose antipsychotic treatment. These methods have confirmed findings previously suspected from clinical experience and suggested by animal models. Scherer et al (1994) showed that $\mathrm{D}_{2}$ receptor occupancy differs between patients with and without extrapyramidal side-effects. Dopamine PET studies have shown that a receptor occupancy of $65 \%$ or higher is needed to achieve an antipsychotic effect, but exceeding $72 \%$ causes hyperprolactinaemia and over $78 \%$ leads to extrapyramidal side-effects (Tauscher \& Kapur, 2001). This translates into the clinically useful concept of response to low-dose antipsychotic treatment, and has allowed approximate dose

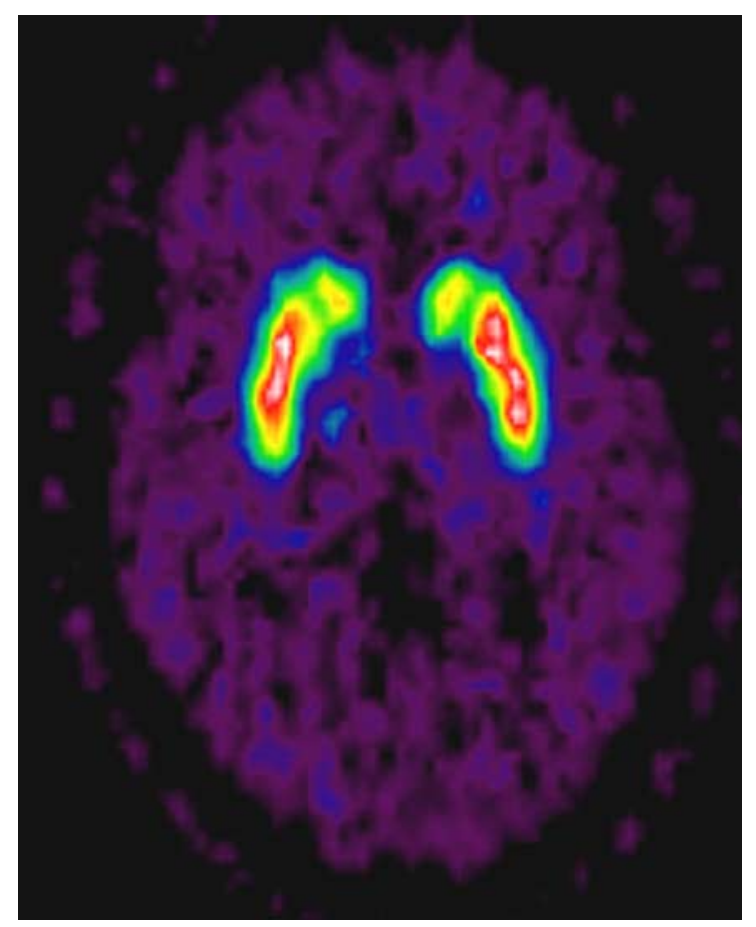

(a)

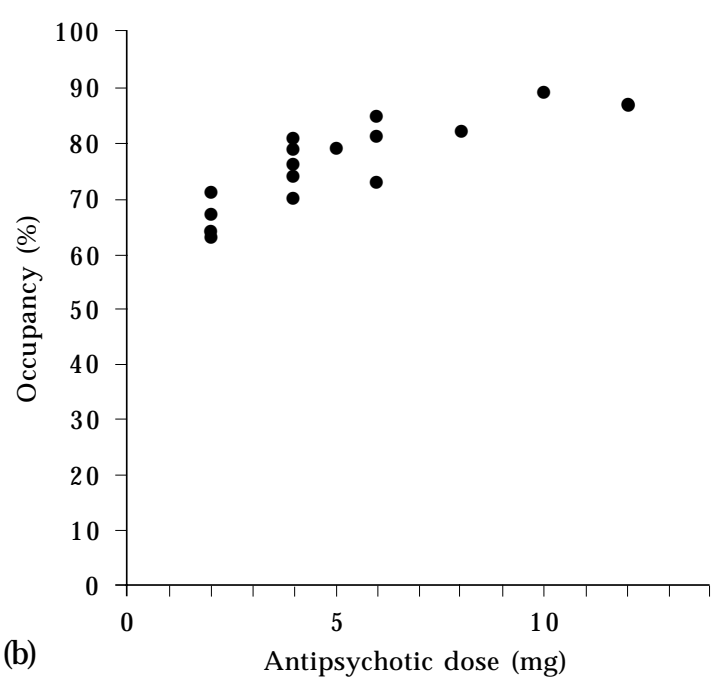

Fig. 2 (a) PET image showing dopamine receptor binding in the basal ganglia; (b) measurements of $\mathrm{D}_{2}$ receptor occupancy made using PET illustrate how occupancy increases with increasing antipsychotic dose. With permission of Professor P. Grasby. Part (a) appears in colour in the online version of this article (accessible via http://apt. repsych.org). 
equivalence between alternative antipsychotics to be calculated (e.g. $5 \mathrm{mg}$ haloperidol per day = risperidone $2 \mathrm{mg}=$ olanzapine $7.5 \mathrm{mg}$; Kapur et al, 1999). Gold et al (1991) demonstrated that neuroimaging can help to predict other side-effects such as tardive dyskinesia. Patients with tardive dyskinesia had significantly smaller ventricle-tobrain ratios than controls.

Recent studies expand on this and help the clinician in the selection of drugs as well as of dose. Increasingly, different side-effect profiles are emerging between the various atypical antipsychotics, and again PET and SPET can illustrate the differing receptor-affinity profiles, allowing the clinician to predict the likelihood of specific sideeffects and tailor drug selection accordingly.

\section{Relapse prediction}

Neuroimaging, in combination with other factors, may highlight the likelihood of relapse. For example, from a host of potential relapse predictors following first-episode schizophrenia or schizoaffective disorder, one of the few positive indicators was hippocampal volume (Robinson et al, 1999).

\section{Identifying risk factors}

Within groups of patients in their first psychotic episode, significant deficits have been found in cortical grey matter, temporal lobe grey matter and whole brain volume as well as significant enlargement of the lateral and third ventricles. As these occur in both treatment-naïve and minimally treated individuals (Fannon et al, 2000), it is likely that at least some abnormalities were present before the onset of frank psychosis. Furthermore, unaffected relatives of individuals with schizophrenia show many of the volumetric abnormalities seen in people with the disorder, although generally to a lesser degree (Lawrie et al, 1999). The same applies to individuals with prodromal signs who are at high risk of developing psychosis. Within this at-risk group, the severity of the abnormalities may be greater in those who develop psychosis than in those who do not (Pantelis et al, 2003). In addition, the development of psychosis in vulnerable individuals appears to be associated with progressive changes in baseline MRI abnormalities (Pantelis et al, 2003; Job et al, 2004). These observations raise the possibility that neuroimaging may provide a means of identifying the subgroup within a population at high risk who are especially likely to develop psychosis. This would be of great clinical utility. Consequently, any clinical intervention intended to reduce the risk of transition has to be applied to the entire group, raising ethical concerns about unnecessary treatment (McGuire, 2002).

Since disturbances of regional brain function may be evident before macroscopic loss of grey matter, functional imaging may be a more sensitive means of detecting such differences than structural imaging. However, to date there have been few studies of this type and various neuroimaging techniques (e.g. fMRI, PET, psychopharmacological challenge such as tryptophan depletion) are being adapted to explore this.

\section{Future directions}

As already mentioned, there are practical problems in using generalised findings from research to interpret results for individual patients. A key issue for many research studies in schizophrenia is that neuroimaging differences are usually quantitative rather than qualitative and are evident at the group rather than the individual level. A major objective of future work will be to develop a way of using data from a single patient to inform clinical assessment and management. In a move in this direction, Kawasaki et al (2002) has used standard clinical MRI scans and a multivariate analysis technique to examine which patterns of grey matter changes differentiate best between groups of individuals with schizophrenia and with schizotypal disorder. They were able to separate the scans of individual patients from those of controls, and the scans of patients with schizophrenia from those with schizotypal disorder, with a sensitivity of $76-88 \%$.

Neuromorphometry, involving the manual selection of a particular region of interest and measurement of the overall size or volume of that structure, is the basis of much research. Newer approaches also examine shape, which can change without major alterations in size or volume. Csernansky et al (1998) showed that, although overall and regional brain volume is smaller in people with schizophrenia than in controls, there are many confounding variables such as individuals' height and gender. However, they found that measuring the shape of the hippocampus provided a more robust means of differentiating patients from controls (successful in about $70 \%$ of cases). Combining shape measures from more than one region (e.g. the hippocampus and pulvinar, a part of the thalamus) appears to increase the sensitivity of this approach (Csernansky et al, 2004).

Broadening the phenotype beyond strict DSM or ICD schizophrenia may help to reveal more primary mechanisms, allowing more probabilistic predictions for individual clinical patients. The study of intermediate phenotypes (identified 
symptomatically or genetically) is shedding light on the role of global and regionally specific dysfunction (Callicott, 2003), so the future holds promise that a risk profile for individual patients may be defined as advances tie fMRI abnormalities to gene function. Already, relationships between gene function and neuroimaging results have been seen in small samples of individuals with intermediate phenotypes whose behaviour or neuropsychological profile does not necessarily differ from those of healthy controls, emphasising the potential power of such an approach at last to begin giving us useful information about genetic variation in individuals (Hariri \& Weinberger, 2003).

Attempts are also being made to exploit the potential statistical power of combining large numbers of scan datasets derived from both research and clinical practice (Governing Council of the Organisation for Human Brain Mapping, 2001). By comparing the results of such analyses with data from individual patient scans, some of the more subtle abnormalities seen in research findings might be more robustly applicable to individual clinical cases.

\section{The wider context}

Despite the best efforts of voluntary sector and professional groups, individuals with schizophrenia are still subject to discrimination, stereotyping and stigma. Patients and their families often hesitate in or avoid seeking help. Neuroimaging can help to counter this by making otherwise invisible and intangible internal processes easier to see and conceptualise externally. This can help to reduce the sense of personal blame and guilt, allowing patients to ask for and receive help and treatment without shame. As Insel comments,

We already have some pretty effective treatments... The frustrating thing is that even though we know how to treat many of these disorders, there are still many, many people ... who are not getting treatment. There's a gap between what we know how to do and what actually happens in the real world. ... Part of [making sure that these treatments are delivered] - a very important part - involves reducing the stigma of mental disease' (Neuroscience Quarterly, 2003).

Although clearly not a justification in itself, we have found that neuroimaging can help reduce this gap for patients, and it is hard to exaggerate the impact that a brain scan image has in an increasingly medicalised society driven by visual media.

Reintegrating psychiatric practice into 'mainstream medicine' is appealing to many, and in its broad application neuroimaging may help this process. However, there is often the temptation to think that neuroimaging is only of use if one concentrates exclusively on biological theories of psychopathology. We suggest it has a role in broader models too, for example in stress-vulnerability concepts of psychosis, where it can inform on events in the earliest stages (Pantelis et al, 2003), or in illuminating the nature of subtle neurodevelopmental abnormalities acting as predisposing factors for subsequent illness (Dazzan et al, 2004). It is encouraging to see neuroimaging taking this broad approach, and the manner in which it can be adapted to complement other approaches (e.g. using genetics and neuropsychology) means that it is likely to teach us more about schizophrenia and become even more clinically relevant.

In summary, it seems that the most promising areas in which research imaging will translate into clinically useful scanning are likely to be: baseline MRI at first episode, in order to exclude other disorders and to predict outcome; serial scanning during the first few years of psychosis, to categorise rate of progression and thus predict outcome; and scanning at-risk individuals to facilitate estimation of the risk of transition to full-blown psychosis and so decide whom to treat.

\section{References}

American Psychiatric Association (2004) Practice Guideline for the Treatment of Patients with Schizophrenia (2nd edn). Arlington, VA: APA. http://www.psych.org/ psych_pract/treatg/pg/Practice\%20Guidelines8904/ Schizophrenia_2e.pdf

Cahn, W., Pol, H. E., Lems, E. B., et al (2002) Brain volume changes in first-episode schizophrenia: a 1-year followup study. Archives of General Psychiatry, 59, 1002-1010.

Cahn, W., van Haren, N. E. M., Pol, H. E., et al (2004) Progressive brain volume changes in the first year of illness predict five-year outcome of schizophrenia. Schizophrenia Research, 67 (suppl. 1), 25.

Callicott, J. H. (2003) An expanded role for functional neuroimaging in schizophrenia. Current Opinion in Neurobiology, 13, 256-260.

Csernansky, J. G., Joshi, S., Wang, L., et al (1998) Hippocampal morphometry in schizophrenia by high dimensional brain mapping. Proceedings of the National Academy of Sciences of the United States of America, 95, 11406-11411.

Csernansky, J. G., Schindler, M. K., Splinter, N. R., et al (2004) Abnormalities of thalamic volume and shape in schizophrenia. American Journal of Psychiatry, 161, 896902.

Connolly, M. \& Kelly, C. (2005) Lifestyle and physical health of people with schizophrenia. Advances in Psychiatric Treatment, 11, 125-132.

Dazzan, P., Morgan, K. D., Orr, K. G., et al (2004) The structural brain correlates of neurological soft signs in AESOP first-episode psychoses study. Brain, 127, 143153.

DeLisi, L. E., Stritzke, P. H., Holan, V., et al (1991) Brain morphological changes in 1st episode cases of schizophrenia: are they progressive? Schizophrenia Research, 5, 206-208. 
Dequardo, J. R., Tomori, O., Brunberg, J. A., et al (1997) Does neuroanatomy predict ECT response? Progress in Neuropsychopharmacology and Biological Psychiatry, 21, 1339-1352.

Falkai, P. (1996) Differential diagnosis in acute psychotic episode. International Clinical Psychopharmacology, 11 (suppl. 2), 13-17.

Fannon, D., Chitnis, X., Doku, V., et al (2000) Features of structural brain abnormality detected in first-episode psychosis. American Journal of Psychiatry, 157, 1829-1834.

Gold, J. M., Egan, M. F., Kirch, D. G., et al (1991) Tardive dyskinesia: neuropsychological, computerized tomographic, and psychiatric symptom findings. Biological Psychiatry, 30, 587-599.

Gopal, Y. V. \& Variend, H. (2005) First-episode schizophrenia: review of cognitive deficits and cognitive remediation. Advances in Psychiatric Treatment, 11, 38-44.

Governing Council of the Organisation for Human Brain Mapping (2001) Neuroimaging databases. Science, 292, 1673-1676.

Hariri, A. R. \& Weinberger, D. R. (2003) Imaging genomics. British Medical Bulletin, 65, 259-270.

Ho, B. C., Andreasen, N. C., Nopoulos, P., et al (2003) Progressive structural brain abnormalities and their relationship to clinical outcome: a longitudinal magnetic resonance imaging study early in schizophrenia. Archives of General Psychiatry, 60, 585-594.

Job, D., Whalley, H. C., Johnstone, E. C., et al (2004) Grey matter changes as people at high risk develop schizophrenia. Schizophrenia Research, 67, 26.

Johnstone, E. C., Crow, T. J., Frith, C. D., et al (1976) Cerebral ventricular size and cognitive impairment in chronic schizophrenia. Lancet, 2, 924-926.

Kapur, S., Zipursky, R. B. \& Remington, G. (1999) Clinical and theoretical implications of 5- $\mathrm{HT}_{2}$ and $\mathrm{D}_{2}$ receptor occupancy of clozapine, risperidone, and olanzapine in schizophrenia. American Journal of Psychiatry, 156, 286293.

Kapur, S., Zipursky, R., Jones, C., et al (2000) Relationship between dopamine $\mathrm{D}(2)$ occupancy, clinical response, and side-effects: a double-blind PET study of first-episode schizophrenia. American Journal of Psychiatry, 157, 514520.

Kasai, K., McCarley, R. W., Salisbury, D. F., et al (2004) Cavum septi pellucidi in first-episode schizophrenia and first-episode affective psychosis: an MRI study. Schizophrenia Research, 71, 65-76.

Kawasaki, Y., Suzuki, M., Nohara, S., et al (2002) Can brain morphological changes be of diagnostic value for psychiatric disorders? A voxel-based morphometric approach. Neuroimage, 16 (suppl. 1), 37-38.

Kerwin, R. W. \& Bolonna, A. (2005) Management of clozapineresistant schizophrenia, Advances in Psychiatric Treatment, 11, 101-106.

Konicki, P. E., Kwon, K. Y., Steele, V., et al (2001) Prefrontal cortical sulcal widening associated with poor treatment response to clozapine. Schizophrenia Research, 48, 173-176.

Lauriello, J., Mathalon, D. H., Rosenbloom, M., et al (1998) Association between regional brain volumes and clozapine response in schizophrenia. Biological Psychiatry, 43, 879886.

Lawrie, S. M., Abukmeil, S. S., Chiswick, A., et al (1997) Qualitative cerebral morphology in schizophrenia: a magnetic resonance imaging study and systematic literature review. Schizophrenia Research, 25, 155-166.

Lawrie, S. M., Whalley, H., Kestelman, J. N., et al (1999) Magnetic resonance imaging of brain in people at high risk of developing schizophrenia. Lancet, 353, 30-33.

Leask, S. J. (2004) Environmental influences in schizophrenia: the known and the unknown. Advances in Psychiatric Treatment, 10, 323-330.

Lubman, D. I., Velakoulis, D., McGorry, P. D., et al (2002) Incidental radiological findings on brain magnetic resonance imaging in first-episode psychosis and chronic schizophrenia. Acta Psychiatrica Scandinavica, 106, 331336.
McCreadie, R. G. (2004) Editorial: Schizophrenia revisited. Advances in Psychiatric Treatment, 10, 321-322.

McGuire, P. K. (2002) Prodromal intervention: the need for evaluation. Journal of Mental Health, 11, 469-470.

McGuire, P. K. \& Matsumoto, K. (2004) Functional neuroimaging in mental disorders. World Psychiatry, 3, 6-11.

Neuroscience Quarterly (2003) Comments. An interview with Thomas Insel, Director of the National Institute of Mental Health. Neuroscience Quarterly, Spring. http://web.sfn. org/content/Publications/NeuroscienceNewsletter/ 2003spring/comments.html.

Pantelis, C., Velakoulis, D., McGorry, P. D., et al (2003) Neuroanatomical abnormalities before and after onset of psychosis: a cross-sectional and longitudinal MRI comparison. Lancet, 361, 281-288.

Robinson, D., Woerner, M. G., Alvir, J. M., et al (1999) Predictors of relapse following response from a first episode of schizophrenia or schizoaffective disorder. Archives of General Psychiatry, 56, 241-247.

Rowlands, P. (2004) The NICE schizophrenia guidelines: the challenge of implementation. Advances in Psychiatric Treatment, 10, 403-412.

Saykin, A. J., Gur, R. C., Gur, R. E., et al (1991) Neuropsychological function in schizophrenia. Selective impairment in memory and learning. Archives of General Psychiatry, 48, 618-624.

Scherer, J., Tatsch, K., Schwarz, J., et al (1994) $\mathrm{D}_{2}$-dopamine receptor occupancy differs between patients with and without extrapyramidal side-effects. Acta Psychiatrica Scandinavica, 90, 266-268.

Schröder, J., Geider, F. J. \& Sauer, H. (1993) Can computerised tomography be used to predict early treatment response in schizophrenia? British Journal of Psychiatry, 163 (suppl. 21), 13-15

Singh, S. P. \& Fisher, H. L. (2005) Early intervention in psychosis: obstacles and opportunities. Advances in Psychiatric Treatment, 11, 71-78.

Smith, G. N., Flynn, S. W., Kopala, L. C., et al (1997) A comprehensive method of assessing routine CT scans in schizophrenia. Acta Psychiatrica Scandinavica, 96, 395-401.

Stern, R. G., Kahn, R. S. \& Davidson, M. (1993) Predictors of response to neuroleptic treatment in schizophrenia. Psychiatric Clinics of North America, 16, 313-338.

Tauscher, J. \& Kapur, S. (2001) Choosing the right dose of antipsychotics in schizophrenia: lessons from neuroimaging studies. CNS Drugs, 15, 671-678.

van Os, J., Fahy, T. A., Jones, P., et al (1995) Increased intracerebral cerebrospinal fluid spaces predict unemployment and negative symptoms in psychotic illness. A prospective study. British Journal of Psychiatry, 166, 750758.

Wassink, T. H., Andreasen, N. C., Nopoulos, P., et al (1999) Cerebellar morphology as a predictor of symptom and psychosocial outcome in schizophrenia. Biological Psychiatry, 45, 41-48.

Weinberger, D. \& Hirsch, S. (2003) Schizophrenia (2nd edn). Oxford: Blackwell Science.

Zipursky, R. B., Lambe, E. K., Kapur, S., et al (1998a) Cerebral gray matter volume deficits in first episode psychosis. Archives of General Psychiatry, 55, 540-546.

Zipursky, R. B., Zhang-Wong, J., Lambe, E. K., et al (1998b) MRI correlates of treatment response in first episode psychosis. Schizophrenia Research, 30, 81-90.

\section{MCQs}

1 Johnstone et al first used neuroimaging to demonstrate ventriculomegaly in schizophrenia in:

a the $1950 \mathrm{~s}$

b the 1960 s

c the $1970 \mathrm{~s}$

d the 1980 s

e the 1990s. 
2 The percentage of patients with psychosis who have an abnormality that accounts for their symptoms detected in a routine radiological MRI scan is:

a zero

b up to $10 \%$

c up to $30 \%$

d up to $50 \%$

e more than $50 \%$.

3 Recent neuroimaging research suggests that:

a there are no MRI abnormalities prior to the first episode of schizophrenia

b there are no MRI abnormalities in the unaffected relatives of patients with schizophrenia

c some of the MRI abnormalities in patients with schizophrenia may progress over time

d prior to the first episode of schizophrenia some of the MRI abnormalities may progress over time

e all of the MRI abnormalities in patients with schizophrenia progress over time.

4 The percentage of patients with psychosis who have abnormalities of any kind on an MRI scan detectable in a routine radiological assessment is:

a up to $12 \%$

b up to $30 \%$

b up to $40 \%$

d up to $50 \%$

e more than $50 \%$
5 The lowest level of striatal $D_{2}$ receptor occupancy required for a therapeutic response to most antipsychotic drugs is:

$\begin{array}{ll}\text { a } & 45 \% \\ \text { b } & 55 \% \\ \text { c } & 65 \% \\ \text { d } & 75 \% \\ \text { e } & 85 \%\end{array}$
MCQ answers

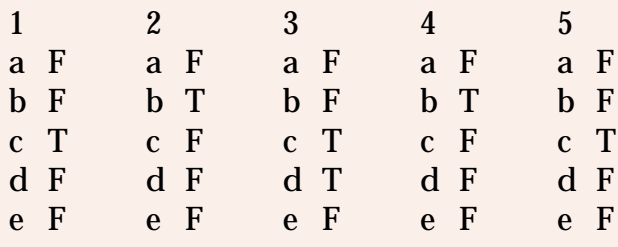

\section{Clinical Evidence Mental Health (third edition)}

Royal College of Psychiatrists/BMJ Publishing Group

This book contains the mental health section extracted from Clinical Evidence, Issue 11 (BMJ Publishing Group), the international source of the best available evidence for effective healthcare. The book presents clear, concise summaries of the current state of the evidence on the prevention and treatment of mental health disorders derived, where possible, from randomised controlled trials and systematic reviews.

This third edition covers 15 different mental health disorders, 27 questions, and over 200 interventions.

- Based on the latest Clinical Evidence search and appraisal results.

- Includes evidence on the short- and long-term treatment of mental health disorders and their complications.

- Assesses drug treatments, non-drug treatments and psychological therapies.

- Uses explicit methodology for selecting which evidence to summarise.

- Categorises interventions according to whether they have been found to be effective or not.

- Presents key messages and structured summaries of filtered evidence.

- Presents the balance between benefits and harms of intervention.

August 2004, Paperback, 210pp, ISBN 190467116 0, Price $£ 14.00$

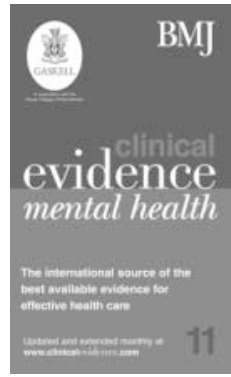

